\title{
Giant abdominal chylolymphatic cyst: Review of Two Rare Cases
}

\author{
Puneet Srivastava*, Richa Jaiman, Surendra Pathak, Juhi Singhal \\ Department of Surgery, S.N. Medical College. Agra. India
}

\begin{abstract}
Mesenteric cysts are rare benign intra peritoneal tumor and more than half of the mesenteric cysts involve the mesentery of the terminal ileum but cyst in mesentery of jejunum and also in retroperitoneum is very rare. A mesenteric cyst is a fluid-filled sac lined with endothelium or mesothelium arising anywhere in the mesentery of the duodenum through the large bowel .We present two cases of Giant chylolymphatic mesenteric cyst one in jejunal mesentery which was very large and other one in retroperitoneum ..In both cases cyst was excised without any complication or recurrence. We would like to emphasize the importance of successful enucleation of the cyst irrespective of its size due to its independent blood supply as opposed to enterogenous cyst which requires bowel resection and anastomosis. We report these cases because of its rare site and size.
\end{abstract}

Keywords: Chylolymphatic, Mesentericcyst, Lymphangioma

\section{Introduction}

First reported in 1907 by the Florentine anatomist Benevieni in an 8-year-old girl[1] A mesenteric cyst is a fluid-filled sac lined with endothelium or mesothelium arising anywhere in the mesentery of the duodenum through the large bowel. They may be filled with serous, chylous, sanguinous or chylolymphatic fluid and may be composed of a single sac, septated or multilocultated[2]. The incidence of mesenteric cysts has been reported to be in the region of 1/100 000-1/200 000 and between $10 \%$ and $30 \%$ of these may be in children although can present at any age [3, 4].Giant mesenteric cysts are even rarer and those containing chylolymphatic fluid are the rarest [2].Clinically mesenteric cysts may present as an asymptomatic abdominal mass, incidental finding during laparotomy for other abdominal conditions, or it may present as an acute abdomen. Mesenteric cysts may cause acute abdomen from the cyst rupture, infection, hemorrhage, intestinal obstruction, and volvulus [5]. USG and computed tomography scan of the abdomen are the investigations of choice [2]. Mesenteric cysts may vary in size from $4 \mathrm{~cm}$ to $30 \mathrm{~cm}$ [5]. Complete surgical excision remains the mainstay for the treatment of the mesenteric cysts with an excellent result [2].

\section{Case Reports}

Case 1: A 2- year- old female presented with swelling in abdomen from 2 month duration. Physical examintion revealed a huge intrabdominal mass filling the whole of the upper abdomen below umblicus.Her biochemical investigations were in the normal range. Ultrasound abdomen revealed a large cystic space occupying lession of size $13 \mathrm{X} 9 \mathrm{cms}$ with multiple septa .CT scan revealed large predominantly cystic mass of size $12.7 \times 10.1 \times 7.5$ cms in abdomen and pelvis with few thickened enhancing septae in upper portion[Figure-1]. Surgical exploration was done which revealed multiple huge cysts $6 \mathrm{~cm}$ distal to dudenojejunal flexure abutting the jejunalmesentry[Figure 2]. Cysts were removed by resection of jejunal segment followed by anastomosis. The diagnosis was confirmed on histopathology which revealed thin cyst wall lined with flat endothelium having clear lymphoid aggregates. Patient was discharged under satisfactory condition.

Case 2: A 4 year female presented with swelling in lower abdomen with off and on pain in abdomen. There was no history of fever vomiting or feature of intestinal obstruction. On examination cystic swelling confined left lower abdomen which was fixed. On CT scan abdomen showed $10 \times 9 \times 9 \mathrm{~cm}$ cyst in left lower retroperitoneum indenting to bladder wall [figure-3]. Surgical exploration was done and cyst was removed as whole from retoperitonem [figure-4]. Surgical outcome was good.

\section{Discussion}

Mesenteric cysts are most commonly solitary and multiloculated, but may occur at multiple positions within the peritoneal cavity. One third of the mesenteric cysts occur in the children younger than 15 years of age and it is reported slightly more often in males [6]. The exact etiology for the development of the mesenteric cysts is not known. The most commonly accepted theory was proposed by Gross, and it is the result of benign 


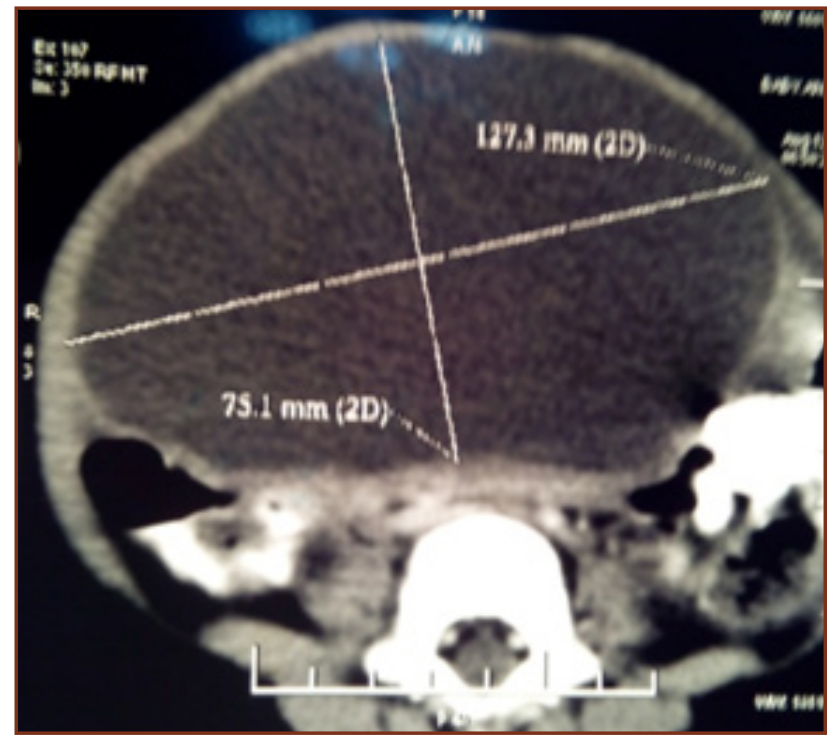

Fig. 1: CT Scan showed large cystic mass of size $12.7 \times 10.1 X 7.5 \mathrm{cms}$ in abdomen with septa.

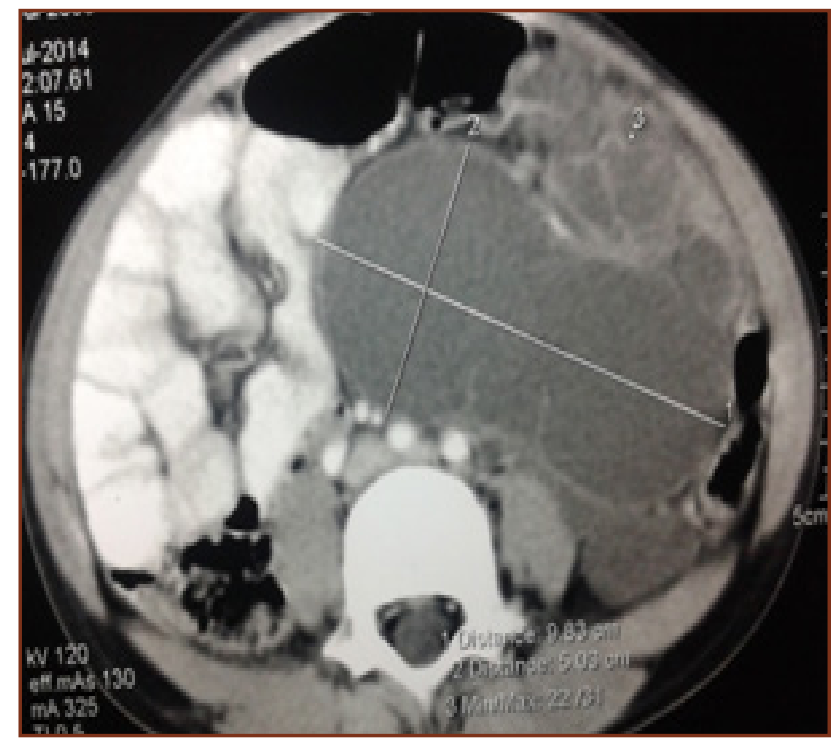

Fig. 3: CT Scan showed large cystic mass of size $10 \times 9.5 \times 9$ cms in retroperitoneum.

proliferation of ectopic lymphatics in the mesentery that lack communication with remainder of the lymphatic system [6] These cysts arise in the sequestrated lymphatic channels or ectopic lymphatic tissue in the small bowel mesentery and enlarge by accumulating both lymph and chyle. The accumulation of lymph and chyle is thought to result from an imbalance between the inflow and outflow of fluid across these channels.[7]. Other cystic lesions in the abdomen that resemble mesenteric cysts are cystic lymphangioma (hygroma), cystic teratoma, ovarian cyst, intestinal duplication cyst, hydatid cyst, etc

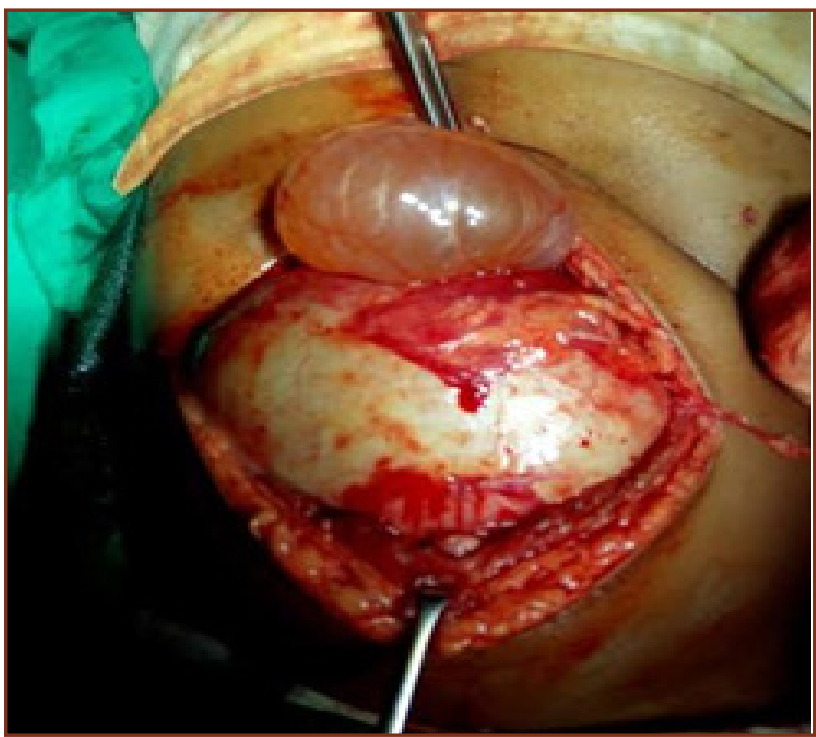

Fig. 2: Intraoperative picture showed multiple other cyst along with larger cyst.

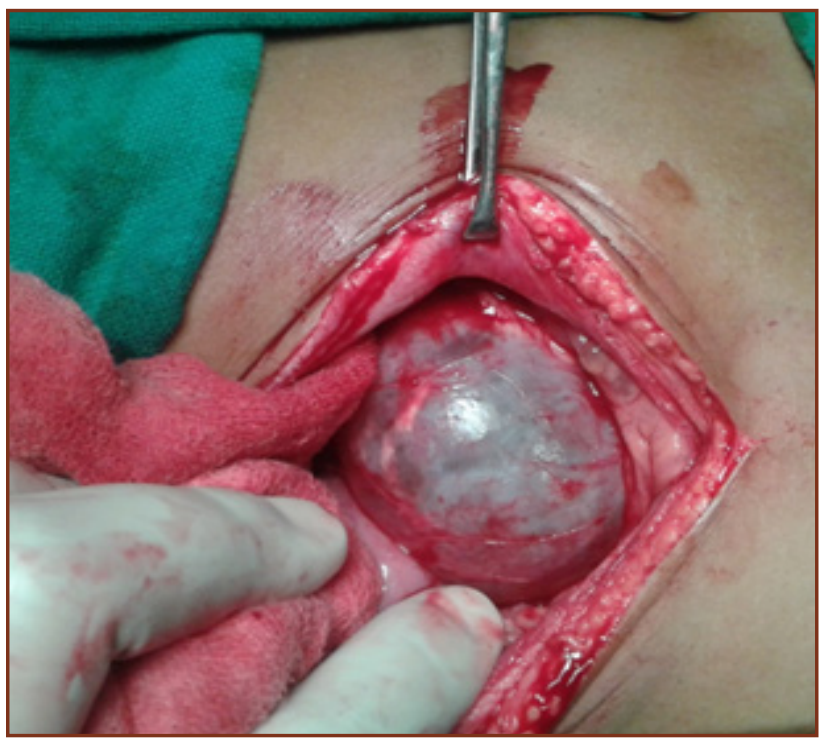

Fig. 4; Intraoperative picture showed larger retroperitoneal cyst

$[2,6]$. Histopathological examination is confirmatory and differentiates chylolymphatic cysts from all these lesions $[2,6]$ Complete surgical excision remains the mainstay for the treatment of the mesenteric cysts with an excellent result[6].In approximately $20 \%$ to $60 \%$ of the cases bowel resection and anastomosis is needed along with the excision of the mesenteric cysts [8]. Excision of the mesenteric cysts may also be accomplished successfully with laparoscopically [8]

This present case the cyst was localized in the mesentery of the jejunum, and it also involved the adjacent ileum. He 
was presented with distention of abdomen. The diagnosis of the cyst was suspected on the USG of the abdomen and was confirmed by CT scan. En bloc complete surgical excision of the cyst involving the mesentery of jejunum along with the involved adjacent bowel was done

\section{Conclusion}

Children with chylolymphatic cyst (mesenteric cyst) involving the mesentery of jejunum may present with features of acute intestinal obstruction. Surgical excision of the mesenteric cyst remains a mainstay of treatment with an excellent result.

\section{References}

1. Mohanty SK, Bal RK, Maudar KK. Mesenteric cyst-an unusual presentation. J PediatrSurg 1998;33:792-3.

2. Rattan K, Vimoj JN, Manish P, et al. Pediatric chylolymphatic mesenteric cyst - a separate entity from cystic lymphangioma: a case series. J Med Case Rep 2009;3:111.

3. Kurtz RJ, Heimann TM, Holt J, et al. Mesenteric and retroperitoneal cysts. Ann Surg 1986;203:109.

4. Bliss DP, Jr, Coffin CM, Bower RJ, et al. Mesenteric cysts in children. Surgery 1994;115:571-7

5. JJ Tan, KK Tan, SP Chew. Mesenteric cysts: an institution experience over 14 years and review of literature. World J Surg. 2009;33:1961-65

6. [1] RR Ricketts.Mesenteric and omental cysts. In: Grosfeld Jay L, O’Neill JA, Fonkalsrud EW, Coran AG, Caldamone AA (eds), Pediatric Surgery. Chapter 89. Mosby Inc. 2006; Vol II:1399-1406.

7. Engel S., Clagett O.T., Harrison Eg., Jr. Chylous cysts of the abdomen. Surgery.1961;50:593-599.

8. NSTran,TLNguyen.Laparoscopicmanagementofabdominal lymphatic cyst in children.J LaparoendoscAdvSurg Tech A. 2012;22:505-07

*Corresponding author:

Dr. Puneet Srivastava, MS MCh, Division of Pdiatric surgery, S.N. Medical College Agra U.P. India

Phone: +919319966783

Email: drpunit979@gmail.com

Financial or other Competing Interests: None. 